\title{
Submitted: \\ 11.05 .2020 \\ Accepted: \\ 05.09.2020 \\ Elasticity of the coracohumeral ligament in patients with frozen shoulder following rotator interval injection: a case series
}

Published:

18.12.2020

\section{Keywords}

frozen shoulder, injection, elastic modulus, ultrasonography, rotator interval

\author{
David McKean ${ }^{1}$, Siok Li Chung ${ }^{1}$, Rebecca te Water Naudé2, \\ Bernard McElroy ${ }^{3}$, Jonathan Baxter ${ }^{4}$, Aniruddha Pendse ${ }^{4}$, \\ Joseph Papanikitas ${ }^{1}$, James Teh², Richard Hughes ${ }^{1}$
}

${ }^{1}$ Radiology Department, Stoke Mandeville Hospital, Buckinghamshire Healthcare NHS Trust, UK

${ }^{2}$ Radiology Department, Nuffield Orthopaedic Centre, Oxford University Hospitals NHS Trust, UK

${ }^{3}$ Orthopaedic Department, Chiltern Hospital, Great Missenden, UK

${ }^{4}$ Orthopaedic Department, Buckinghamshire Healthcare NHS Trust, UK

Correspondence: David McKean, Buckinghamshire Healthcare NHS Trust, Stoke Mandeville Hospital, HP218AL, United Kingdom,e-mail: David.McKean@buckshealthcare.nhs.uk

DOI: $10.15557 / \mathrm{JoU} .2020 .0052$

\begin{abstract}
Aim of the study: To evaluate changes in the elasticity of the coracohumeral ligament in patients with adhesive capsulitis of the shoulder treated with ultrasound-guided rotator interval injections. Methods: Shear wave elastography was used to evaluate elasticity of the coracohumeral ligament in symptomatic and asymptomatic shoulders in the shoulder-neutral position and $30^{\circ}$ external rotation. A total of 24 shoulders were assessed. Symptomatic shoulders were treated with targeted steroid injection via the rotator interval and manipulation under local anaesthetic block. Follow-up assessment of the elasticity of the coracohumeral ligament was obtained at 10 weeks post-injection. Results: In all subjects, the coracohumeral ligament elastic modulus was larger at $30^{\circ}$ external rotation than in the neutral position. In patients with adhesive capsulitis, the coracohumeral ligament thickness and elastic modulus was significantly greater in the symptomatic shoulder in the neutral position and $30^{\circ} \mathrm{ER}$. Treated patients had an excellent response with improved Oxford Shoulder Score and reduced visual analogue scale pain scores. Median Oxford Shoulder Score was 13.5 pre-injection and 34 at 10 weeks postinjection. Median visual analogue scale pain scores measured 8.5 pre-injection, 3.5 at 1 day, 2 at 1 week, and 2.5 at 10 weeks. Improved Oxford Shoulder Score and visual analogue scale pain score was associated with a trend to normalisation of the elastic modus of the coracohumeral ligament. Conclusion: In patients with adhesive capsulitis of the shoulder, shear wave elastography demonstrated the coracohumeral ligament is stiffer in the symptomatic shoulder than in the unaffected shoulder. Treatment with the ultrasound-guided rotator interval injection is associated with improved Oxford Shoulder Score, reduced visual analogue scale pain scores, and reduced stiffness in the coracohumeral ligament.
\end{abstract}

\section{Introduction}

Adhesive capsulitis of the shoulder, also known as frozen shoulder, is a common orthopaedic condition characterised by loss of glenohumeral motion ${ }^{(1)}$. The underlying cause of adhesive capsulitis is not well understood but appears to be characterised by synovial inflammation and capsular fibrosis $^{(2)}$. Several recent studies have implicated the rotator interval and coracohumeral ligament as being critical in the pathology of adhesive capsulitis ${ }^{(3-6)}$. The coracohumeral ligament (CHL) runs from the lateral base of the coracoid process to the lesser and greater tuberosities of the humerus, forming the roof of the rotator interval, and covers the supraspinatus and infraspinatus tendons. The 


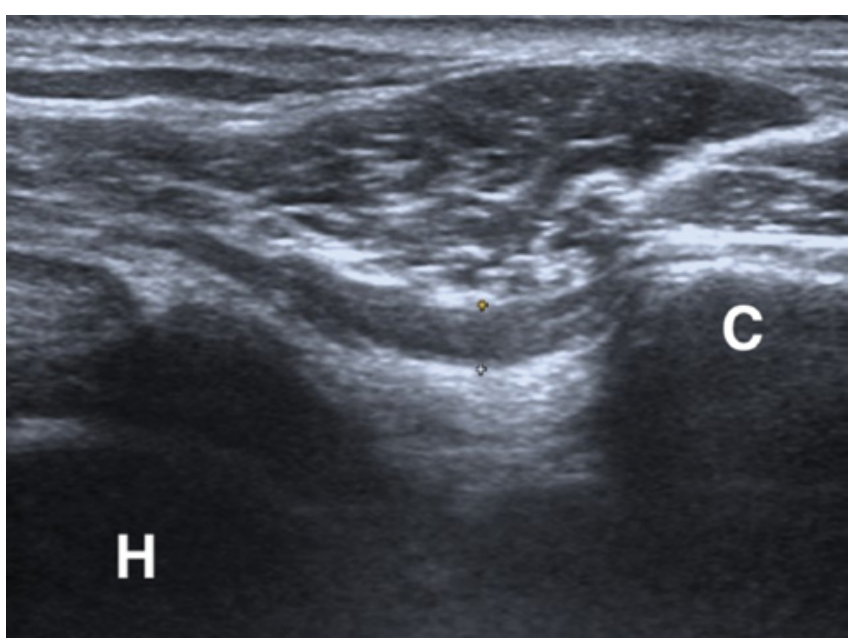

Fig. 1. B-mode US scan shows measurement of coracohumeral ligament thickness in a patient with severe ACS. C-coracoid process, $H$-humeral head

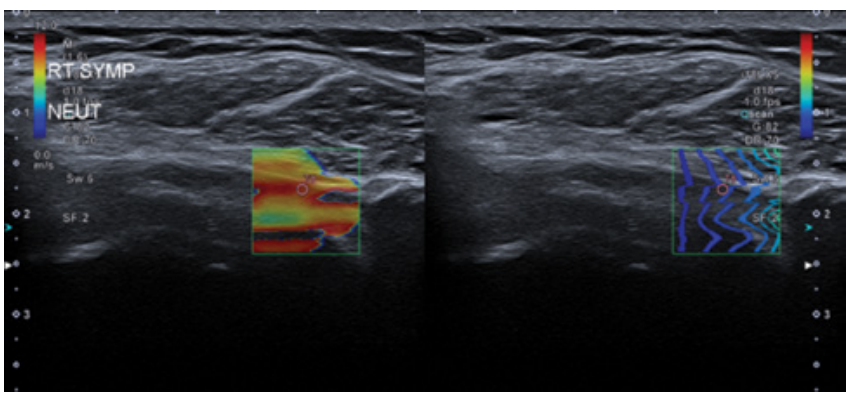

Fig. 2. Measurement of the elastic modus of the coracohumeral ligament using shear wave elastography (SWE)

CHL may play a role in restricting external rotation of the glenohumeral joint ${ }^{(7)}$ and abnormal thickening of this ligament has been reported to be related to restriction of both internal and external rotation ${ }^{(5,8-10)}$. Surgical studies have reported that coracohumeral release is associated with a significant recovery of shoulder range of movement $t^{(5,11,12)}$. Recent studies have also shown that the stiffness of the CHL can be assessed using shear wave elastography ${ }^{(13-15)}$. Shear wave elastography is a relatively new technique to non-invasively provide quantitative information regarding tissue elasticity in real time based on their viscoelastic properties. The ultrasound system propagates shear waves into tissues and assesses their elastic modulus. This has been used in a variety of musculoskeletal conditions including the rotator cuff tendons ${ }^{(16,17)}$, epicondylitis ${ }^{(18)}$, and Achilles $^{(19)}$. Wu et al. reported that in patients with clinical findings consistent with adhesive capsulitis shear wave elastography demonstrated that the CHL was significantly stiffer in the symptomatic shoulder compared with the asymptomatic shoulder ${ }^{(13)}$. Kanazawa et al. have shown that decreased CHL elasticity was strongly associated with decreased shoulder range of movement ${ }^{(14,15)}$. The object of this study was to investigate whether successful treatment of adhesive capsulitis of the shoulder (ACS), treated with ultrasound guided rotator interval injection, was associated with post-injection changes in the elasticity of the CHL as measured using shear wave elastography.

\section{Materials and methods}

The study included patients who were referred from our local shoulder orthopaedic surgeons for consideration for outpatient therapeutic-guided injection over a 4-month period (June 2019 to September 2019).

The inclusion criteria were: a clinical diagnosis of adhesive capsulitis characterised by restriction in range of motion in the affected shoulder, in particular external rotation, failure to respond to conservative management (e.g., physiotherapy, oral analgesia) or blind steroid injections, and the absence of full thickness rotator cuff tear or glenohumeral joint osteoarthrosis. Suitable patients had a pre-procedure ultrasound examination, received manipulation under anterior interval local anaesthetic block procedure as described below, and a post-procedure ultrasound examination at the time of orthopaedic clinic follow up at 10 weeks.

\section{Ultrasonography evaluation}

A musculoskeletal radiology consultant with 10 years of musculoskeletal ultrasound experience performed ultrasonographic assessment of the shoulder rotator cuff tendons, bursa and CHL. The participants were assessed sitting upright or semi-supine. The elbow was held in 90 degrees of flexion with the arm at the side for initial assessment. B-mode ultrasound was performed to assess the thickness of the CHL. A longitudinal image of the CHL was achieved in an axial oblique plane and the thickness of the CHL measured at a $2 \mathrm{~mm}$ distance from the coracoid process (Fig. 1), consistent with previous study methodology ${ }^{(13)}$. SWE assessment was performed in the same position and again with the arm in 30 degrees external rotation, or the maximal degree of external rotation that the patient was able to achieve. The patient's skin was covered in abundant gel, and the probe was placed without applying pressure on the underlying tissues. The patients were asked to hold their breath for 5-10 seconds during SWE measurements to avoid interference from chest wall movement. Elasticity of the CHL was assessed using a ROI with a diameter of $1 \mathrm{~mm}$ at a distance of $2 \mathrm{~mm}$ from the coracoid process, and the elastic modulus was recorded in triplicate. The average of these measurements was used for statistical analysis (Fig. 2).

\section{Injection technique}

A $15-\mathrm{MHz}$ linear array ultrasound transducer (Aplio i700, Cannon, UK), sterile ultrasound gel, two $10 \mathrm{ml}$ syringes, one $5 \mathrm{ml}$ syringe, and a $25 \mathrm{~mm}$ long 23-gauge (green) needle were used.

A pre-injection assessment of the patient's range of motion is performed with the patient sitting, followed by a routine ultrasound examination of the shoulder, in particular assessing for pathology in the rotator cuff, rotator interval pathology, CHL thickening, subacromial subdeltoid effusion or impingement on dynamic imaging. 


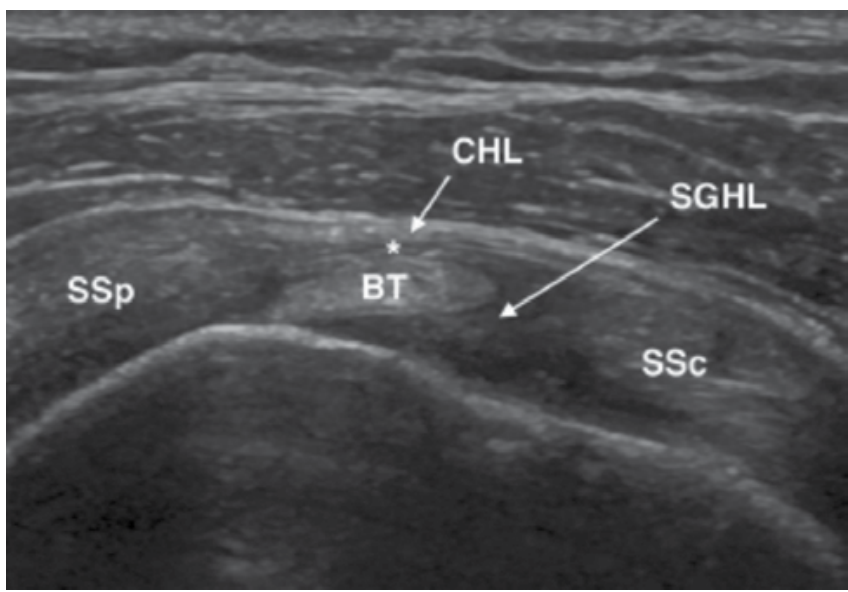

Fig. 3. A transverse ultrasound image of the rotator interval with the long head of biceps tendon (BT) at the centre of the image. The superior glenohumeral ligament (SGHL) is anterior to the biceps tendon and the coracohumeral ligament (CHL) forms the roof of the interval superiorly. The supraspinatus tendon (SSp) lies laterally and subscapularis tendon (SSc) lies medially. The asterisk indicates the target point of the needle for targeted rotator interval injection

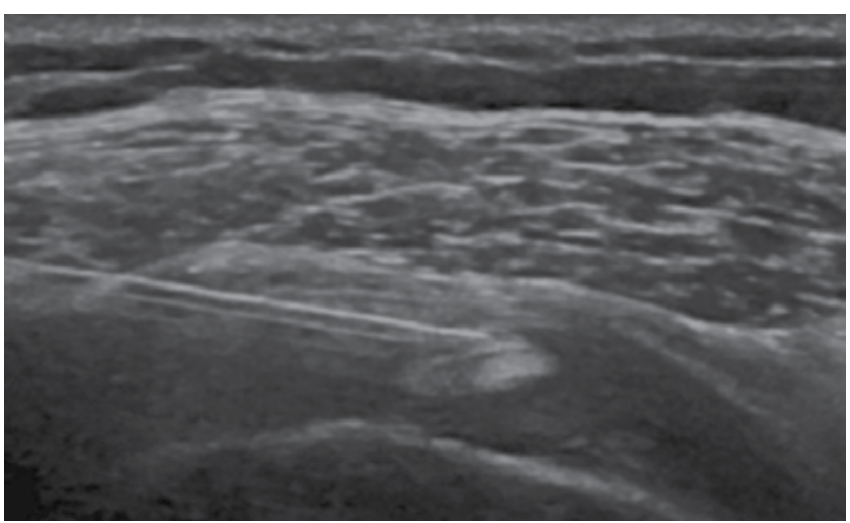

Fig. 4. Transverse ultrasound image of the rotator interval with the biceps tendon at the centre of the image. Using a long axis needle approach, the needle tip lies between the coracohumeral ligament, as it forms the roof of the rotator interval, and the long head of biceps tendon sheath below

If there was sufficient clinical evidence of adhesive capsulitis, as demonstrated by a global reduction in range of motion, especially external rotation, in the absence of rotator cuff pathology, the patient was consented for an ultrasound guided rotator interval injection.

The patient may sit upright or lie semi-supine. The shoulder is slightly extended, and the hand supinated to facilitate visualisation of the rotator interval anteriorly. The transducer is placed over the anterior shoulder and a long-axis view of the rotator interval, with the biceps at the centre of image and supraspinatus and subscapularis to either side, is obtained (Fig. 3). The often thickened CHL is seen draped superiorly over the biceps tendon.

Routine skin antisepsis and subcutaneous infiltration with $1 \%$ lidocaine is performed with a $25 \mathrm{~mm} 23$-gauge needle,

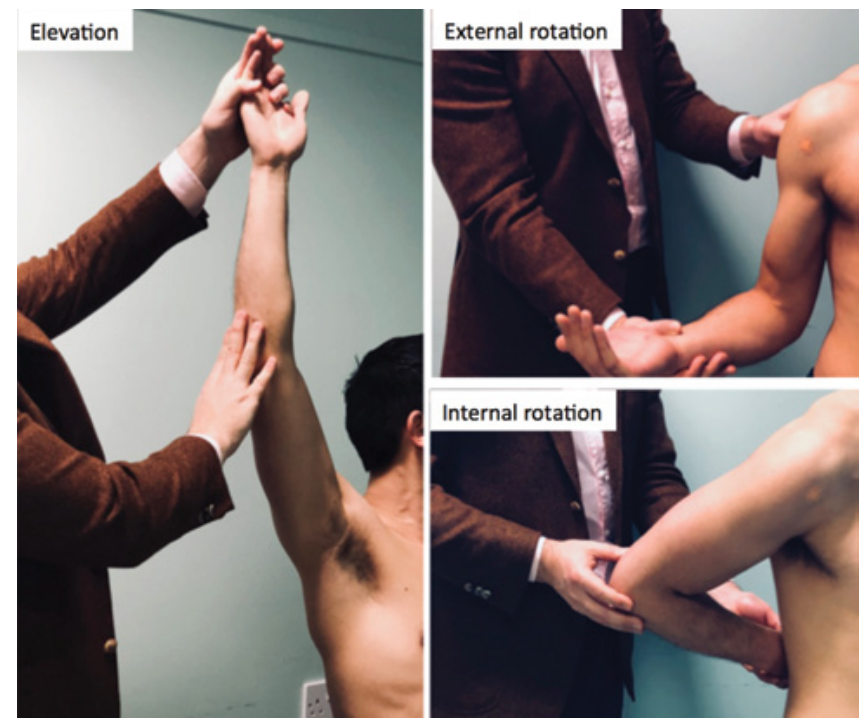

Fig. 5. Shoulder manipulation technique. The patient is asked to demonstrate their range of motion before the injection is performed, and following the procedure is gently helped into elevation, external rotation and internal rotation using a gentle stretching motion

which may then be advanced just superficial to the CHL with further infiltration of $1 \%$ lidocaine. Following this, the needle is advanced deep to the CHL, into the rotator interval, positioned in the biceps tendon sheath between the CHL above and biceps tendon below (Fig. 4), and a $12 \mathrm{ml}$ mixture of $1 \%$ lidocaine and $0.5 \%$ bupivacaine and $40 \mathrm{mg}$ triamcinolone is gently instilled. With correct intrasheath needle positioning, the injected fluid should flow freely away from the needle tip. This may be confirmed using Doppler imaging with a rim of flow surrounding the long head of biceps tendon confirming extension of the injectate into the joint. Following injection, patients are invited to perform active movement of the shoulder. Patients are asked to lift their arm above their head. For internal rotation, patients are asked to place their hand between their shoulder blades. For external rotation, patients are asked to keep their elbow tucked in and turn their supinated hand out as far as they can go. Patients often notice an immediate improvement in their range of movement and are then gently manipulated into a full range of elevation, internal and external rotation under local anaesthetic block (Fig. 5).

Patients were assessed pre-injection with documented pain scores from 0 to 10 on a visual analogue scale (VAS) and the Oxford Shoulder Score (OSS) Questionnaire, a validated 12-item questionnaire for measuring patient perception of shoulder problems, with a possible range of scores from 0 (severe shoulder symptoms) to 48 (no shoulder symptoms) ${ }^{(20)}$. Initial follow-up comprised a VAS pain score at 1 hour, 24 hours, and 2 weeks. Clinical review by the referring orthopaedic surgeon was performed at 2 months post-injection. Long-term follow-up involved a VAS pain score and the OSS Questionnaire at 5 months. Statistical analysis was performed using SPSS Version 20 and GraphPad Prism Version 6.0. The Kolmogorov-Smirnov 
Tab. 1. Cohort demographics

\begin{tabular}{|l|c|}
\hline Parameter & 24 \\
\hline Number of shoulders assessed & 6 male; 6 female \\
\hline Gender & $54(28-72)^{*}$ \\
\hline Age (years) & $12(3-36)^{*}$ \\
\hline Duration of symptoms (months) & * Data are medians, with ranges given in parentheses. \\
\hline
\end{tabular}

Tab. 2. Comparison of coracohumeral ligament ( $\mathrm{CHL}$ ) thickness and elasticity on the symptomatic and asymptomatic sides

\begin{tabular}{|l|c|c|c|}
\hline Parameter & $\begin{array}{c}\text { Asymptomatic } \\
\text { side }(\boldsymbol{n}=\mathbf{1 2})\end{array}$ & $\begin{array}{c}\text { Symptomatic } \\
\text { side }(\boldsymbol{n}=\mathbf{1 2})\end{array}$ & Pvalue $^{\mp}$ \\
\hline $\begin{array}{l}\text { CHL thickness } \\
(\mathbf{m m})\end{array}$ & $1.95(1.1-2.9)^{*}$ & $3.3(2.4-3.7)^{*}$ & 0.00222 \\
\hline $\begin{array}{l}\text { CHL elastic } \\
\text { modulus }(\mathbf{k P a})\end{array}$ & & & \\
\hline Neutral position & $178(116.4-228.1)^{*}$ & $278(194.7-338.4)^{*}$ & 0.00222 \\
\hline $\begin{array}{l}\mathbf{3 0}^{\circ} \text { external } \\
\text { rotation }\end{array}$ & $194(138-246.5)^{*}$ & $298(210-342.2)^{*}$ & 0.00222 \\
\hline OSS & na & $18(7-39)$ & na \\
\hline VAS pain score & na & $8(6-10)$ & na \\
\hline
\end{tabular}

* Data are medians, with ranges given in parentheses. ${ }^{\dagger}$ Symptomatic shoulder assessed at $30^{\circ}$ external rotation or as close to this position as the patient could achieve. ${ }^{\ddagger}$ Comparison between asymptomatic and symptomatic shoulders with use of the Wilcoxon Signed-Rank test calculator. OSS - Oxford Shoulder Score

test of normality was used, and the Wilcoxon test was used to establish whether there was a significant difference $(p<0.05)$ between pre- and post-procedure scores.

Data regarding demographics, duration of symptoms, relevant history, co-morbidities, and other treatments for their shoulder were recorded. The ultrasound findings in the shoulder at the time of the procedure were also documented

\section{Results}

A total of 24 shoulders were assessed pre-treatment (6 men, 6 women, median age 54 years, range $28-72$ years) (Tab. 1). In all subjects, the CHL elastic modulus was observed to be larger at $30^{\circ}$ external rotation than in the neutral position. In our cohort of patients with ACS the CHL thickness was significantly greater in the symptomatic shoulder (median $3.3 \mathrm{~mm}$ vs $1.95 \mathrm{~mm}$ ) and the elastic modulus, measured in the neutral position, of the symptomatic shoulder (mean $269.7 \mathrm{kPa}$, range 194.7-338.4 kPa, median $278 \mathrm{kPa}$ ) was significantly greater than that of the unaffected shoulder (mean $175 \mathrm{kPa}$, range 131-216 kPa, median $178 \mathrm{kPa}$ ) and $30^{\circ}$ external rotation (Tab. 2 and Tab. 3). Several patients had very severe restriction of external rotation on the symptomatic side, and were asked to move as close to $30^{\circ}$ of external rotation as they were able to achieve and assessed in this position.

A total of 12 symptomatic shoulders were treated with targeted ultrasound-guided steroid injection via the rotator interval and shoulder manipulation under local
Tab. 3. Coracohumeral ligament (CHL) thickness and elasticity on the symptomatic and asymptomatic sides in neutral and $30^{\circ}$ external rotation (ER) position

\begin{tabular}{|c|c|c|c|c|c|c|c|c|c|c|c|c|c|c|}
\hline \multirow{11}{*}{ 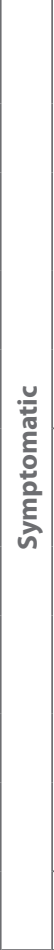 } & \multirow{10}{*}{ 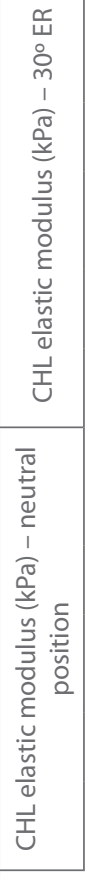 } & 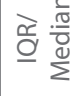 & $\stackrel{\infty}{0}$ & $\begin{array}{l}\tilde{0} \\
\text { Oे }\end{array}$ & $\stackrel{0}{0}$ & $\frac{1}{0}$ & 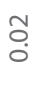 & $\stackrel{m}{\text { mo }}$ & ợ & $\stackrel{\text { Ln }}{\frac{0}{0}}$ & $\stackrel{\text { Oे }}{0}$ & ô. & ס̊ & $\stackrel{\circ}{\circ}$ \\
\hline & & $\stackrel{\stackrel{\Upsilon}{\mathrm{O}}}{ }$ & $\hat{\stackrel{\omega}{\sigma}}$ & $\stackrel{\infty}{\circ}$ & $\stackrel{n}{\sim}$ & 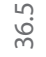 & $\stackrel{\infty}{\wedge}$ & $\begin{array}{l}\text { ఠి } \\
\text {. }\end{array}$ & i் & $\stackrel{\stackrel{\sigma}{m}}{\stackrel{\gamma}{\sigma}}$ & $\stackrel{\bullet}{\circ}$ & $\stackrel{\text { ஸn }}{\text { ¿n }}$ & $\stackrel{m}{\sim}$ & $\begin{array}{l}\dot{\varphi} \\
\dot{m}\end{array}$ \\
\hline & & 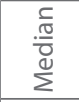 & $\frac{m}{\stackrel{m}{m}}$ & 㞩 & $\stackrel{n ?}{\frac{n}{\sim}}$ & $\stackrel{\infty}{\stackrel{\infty}{N}}$ & $\stackrel{m}{m}$ & $\stackrel{\text { मे }}{m}$ & $\begin{array}{l}\text { ம. } \\
\stackrel{\infty}{\infty} \\
\stackrel{\infty}{0}\end{array}$ & $\stackrel{\text { \} }{\stackrel{D}{\sim}}} &{\begin{array}{l}\text { ¿্ं } \\
\text { ه }\end{array}} &{\begin{array}{l}m \\
\mathscr{\varphi} \\
\grave{m}\end{array}} &{\begin{array}{l}\infty \\
\stackrel{\mathbb{N}}{\text { N}}\end{array}} &{\text { बें }} \\
{\hline} &{ } &{\text { 只 }} &{\stackrel{8}{\stackrel{8}{\complement}}} &{\begin{array}{l}\text { ํ. } \\
\text { 으 }\end{array}} &{\begin{array}{l}8 \\
\infty \\
\infty\end{array}} &{\stackrel{\bullet}{\stackrel{0}{n}}} &{\begin{array}{l}\text { 오․ } \\
\text { nn } \\
\text { nิ }\end{array}} &{\begin{array}{l}\text { 우 } \\
\text { भु }\end{array}} &{\begin{array}{l}\text { ㅇ. } \\
\text { ㅇ. }\end{array}} &{\begin{array}{l}\stackrel{8}{0} \\
m \\
m\end{array}} &{\stackrel{\mathscr{n}}{\sim}} &{\underset{\infty}{\stackrel{0}{0}}} &{\stackrel{\text { ㅁ }}{\text { i }}} &{\frac{\text { 음 }}{\text { in }}} \\
{\hline} &{ } &{\begin{array}{l}\frac{\pi}{\mathbb{J}} \\
\sum\end{array}} &{\begin{array}{l}n \\
\infty \\
\infty \\
0 \\
m\end{array}} &{\text { 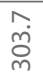 }} &{\stackrel{\circ}{\sim}} &{\text { 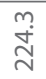 }} &{\begin{array}{l}\text { ô } \\
\text { oे } \\
\text { m }\end{array}} &{\begin{array}{l}\stackrel{0}{\circ} \\
\stackrel{m}{m}\end{array}} &{\begin{array}{l}\infty \\
\stackrel{\infty}{\sim} \\
\stackrel{\infty}{\sim}\end{array}} &{\underset{\substack{n \\
\stackrel{n}{\sim}}}{\sim}} &{\text { ָั }} &{\text { 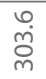 }} &{\stackrel{+}{\stackrel{N}{N}}} &{\text { ঙี }} \\
{\hline} &{ } &{\text { 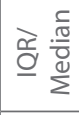 }} &{\frac{0}{0}} &{\overline{0}} &{\stackrel{m}{0}} &{\stackrel{\mathfrak{T}}{\circ}} &{\text { ָै }} &{\text { ¿্. }} &{\stackrel{1}{0}} &{\bar{c}} &{\stackrel{0}{0}_{0}^{0}} &{\stackrel{\text { ֻ̊ }}{\text { ஸे }}} &{\text { : }} &{\frac{\infty}{\sigma}} \\
{\hline} &{ } &{\stackrel{\stackrel{\mathscr{O}}{\mathrm{O}}}{ }} &{\stackrel{\operatorname{Ln}}{\underset{f}{f}}} &{\stackrel{\circ}{m}} &{\overline{6}} &{\stackrel{\bullet}{\stackrel{\sim}{N}}} &{\stackrel{\leftrightarrow}{0}^{\circ}} &{\text { 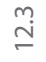 }} &{\text { }} &{\hat{\circ}} &{\stackrel{\circ}{\circ}} &{\hat{0}} &{\stackrel{\circ}{\circ}} &{\text { نْ }} \\
{\hline} &{ } &{\begin{array}{l}\frac{c}{\pi} \\
\frac{\pi}{\pi} \\
\sum \\
\sum\end{array}} &{\text { 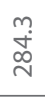 }} &{\begin{array}{l}\infty \\
\stackrel{\infty}{\sim} \\
\stackrel{\infty}{N}\end{array}} &{\text { 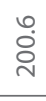 }} &{\text { 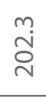 }} &{\text { oे }} &{\text { ָ̊. }} &{\begin{array}{l}\stackrel{0}{0} \\
\stackrel{\infty}{\sim} \\
\sim\end{array}} &{\text { 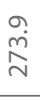 }} &{\begin{array}{l}\text { ¿̊. } \\
\text { ஷे }\end{array}} &{\frac{m}{\grave{n}}} &{\stackrel{m}{\frac{m}{\sim}}} &{\stackrel{0}{\circ}} \\
{\hline} &{ } &{\text { 只 }} &{\stackrel{\infty}{\sim}} &{\stackrel{m}{I}} &{\stackrel{\stackrel{\sim}{*}}{\sim}} &{\stackrel{ }{\Sigma}} &{\text { 守 }} &{\hat{\sim}} &{\stackrel{\infty}{\grave{N}}} &{\frac{a}{m}} &{\stackrel{\mathscr{n}}{\forall}} &{\begin{array}{l}\infty \\
\stackrel{\infty}{\sim}\end{array}} &{\bar{\infty}} &{\begin{array}{l}\infty \\
\stackrel{\infty}{\sim}\end{array}} \\
{\hline} &{ } &{\text { 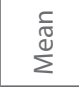 }} &{\begin{array}{l}\infty \\
\infty \\
\stackrel{\infty}{N}\end{array}} &{\begin{array}{l}\stackrel{+}{0} \\
\stackrel{\infty}{\sim}\end{array}} &{\stackrel{\infty}{\text { N }}} &{\text { 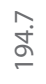 }} &{\text { 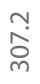 }} &{\begin{array}{l}+ \\
\infty \\
m \\
m\end{array}} &{\text { 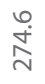 }} &{\stackrel{\infty}{\stackrel{\infty}{\sim}}} &{\text { ৯্ণ }} &{\text { 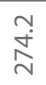 }} &{\begin{array}{l}\text { oे } \\
\infty \\
\stackrel{\nu}{N}\end{array}} &{\begin{array}{l}m \\
0 \\
0 \\
0 \\
\text { m }\end{array}} \\
{\hline} &{\overrightarrow{\underline{U}}} &{ } &{\stackrel{m}{m}} &{m} &{\hat{m}} &{\stackrel{\infty}{m}} &{\hat{m}} &{\hat{m}} &{m} &{\stackrel{\sim}{\sim}} &{m} &{\bar{m}} &{\stackrel{\leftrightarrow}{m}} &{\stackrel{m}{m}} \\
{\hline} &{\begin{array}{l}\text { 品 } \\
\text { ○े } \\
\text { । }\end{array}} &{\text { 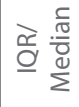 }} &{\stackrel{\sim}{m}} &{\frac{0}{\circ}} &{\stackrel{n}{0}} &{\stackrel{\mathscr{N}}{0}} &{\frac{0}{\pi}} &{\frac{\circ}{\circ}} &{\stackrel{\sim}{\leftarrow}} &{\frac{1}{0}} &{\text { ָ̃ }} &{\frac{N}{0}} &{\stackrel{8}{\circ}} &{\text { ợ }} \\
{\hline} &{\frac{\widehat{\sigma}}{2}} &{\stackrel{\stackrel{\circ}{\mathrm{d}}}{ }} &{\text { กั }} &{\begin{array}{l}\infty \\
\stackrel{\infty}{\circ} \\
\stackrel{\text { }}{ }\end{array}} &{\text { in }} &{\frac{\text { Ln? }}{\frac{\gamma}{\gamma}}} &{\bar{N}} &{ \pm} &{\stackrel{\circ}{\stackrel{\sim}{\sim}}} &{\bar{\infty}} &{\stackrel{ナ}{+}} &{\text { 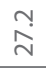 }} &{\stackrel{+}{=}} &{\stackrel{\infty}{\stackrel{\infty}{\perp}}} \\
{\hline} &{\begin{array}{l}\frac{2}{5} \\
\frac{7}{0} \\
\stackrel{\circ}{\xi}\end{array}} &{\text { 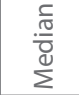 }} &{\text { ma. }} &{\text { 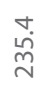 }} &{\begin{array}{l}\text { 으 } \\
\text { gे }\end{array}} &{\text { ڤิڤ }} &{\begin{array}{l}\text { ָु } \\
\text { Jै }\end{array}} &{\stackrel{\hat{n}}{\stackrel{m}{n}}} &{\stackrel{\stackrel{n}{m}}{\stackrel{n}{N}}} &{\text { 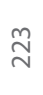 }} &{\text { 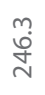 }} &{\begin{array}{l}\text { ָे } \\
\text { N. }\end{array}} &{\text { 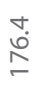 }} &{\text { ஜே }} \\
{\hline} &{\begin{array}{l}\stackrel{u}{\breve{n}} \\
\frac{\tilde{C}}{0}\end{array}} &{\text { 只 }} &{\text { ֶָ }} &{\stackrel{\operatorname{Ln}}{\underline{-}}} &{\text { ָָ }} &{\begin{array}{l}\stackrel{L}{\infty} \\
\infty\end{array}} &{\widehat{\infty}} &{\text { ભ̆ }} &{\text { 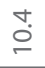 }} &{\stackrel{m}{\stackrel{m}{\sim}}} &{\stackrel{\infty}{-}} &{\underset{0}{+}} &{\overline{i n}} &{\underset{\infty}{\infty}} \\
{\hline \text { 䜦 }} &{\stackrel{\vec{I}}{\cup}} &{\sum_{\substack{\mathbb{N} \\
\sum}}^{\stackrel{0}{\pi}}} &{\text { 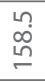 }} &{\stackrel{n}{\stackrel{n}{n}}} &{\begin{array}{l}\stackrel{\sim}{\infty} \\
\stackrel{\infty}{\sigma}\end{array}} &{\begin{array}{l}\text { ํㅜ } \\
\stackrel{0}{0} \\
-\end{array}} &{\begin{array}{l}\stackrel{g}{m} \\
\stackrel{y}{\leftarrow}\end{array}} &{\stackrel{\infty}{m}} &{\stackrel{\bar{N}}{\stackrel{\pi}{N}}} &{\begin{array}{l}\infty \\
\stackrel{\infty}{\sim} \\
\stackrel{\sim}{v}\end{array}} &{\begin{array}{l}\text { In } \\
\stackrel{\leftrightarrow}{\sim} \\
\sim\end{array}} &{\stackrel{i}{\stackrel{i}{N}}} &{\text { ๙ே. }} &{\begin{array}{l}0 \\
\stackrel{1}{\alpha} \\
\end{array}} \\
{\hline \text { 흘 }} &{\text { 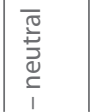 }} &{\text { 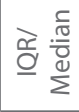 }} &{\stackrel{N}{\check{0}}} &{\text { 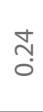 }} &{\frac{\ln }{\dot{0}}} &{\text { ָָ }} &{\text { o̊ }} &{\frac{\sigma}{0}} &{\stackrel{\operatorname{Ln}}{\circ}} &{\frac{\pi}{0}} &{\text { O̊. }} &{\text { ָָ }} &{\text { ס̊. }} &{\stackrel{\mathscr{n}}{\text { ִָ }}} \\
{\hline} &{ } &{\stackrel{\stackrel{q}{0}}{\underline{d}}} &{\stackrel{0}{ }} &{\frac{\text { In }}{i n}} &{\begin{array}{l}\text { oे } \\
\stackrel{\infty}{\sim}\end{array}} &{\begin{array}{l}\infty \\
\dot{m} \\
\dot{m}\end{array}} &{\text { 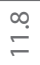 }} &{\stackrel{\sim}{\sim}} &{\begin{array}{l}0 \\
\stackrel{m}{0}\end{array}} &{\begin{array}{l}\stackrel{0}{\sim} \\
\stackrel{\text { D }}{ }\end{array}} &{\text { 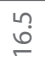 }} &{\begin{array}{l}\text { Oे } \\
\text {. }\end{array}} &{\stackrel{+}{\dot{m}}} &{\stackrel{\circ}{\stackrel{\sim}{\gamma}}} \\
{\hline} &{\text { 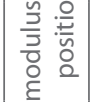 }} &{\text { 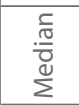 }} &{\stackrel{\check{m}}{\check{m}}} &{\text { 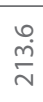 }} &{\stackrel{\infty}{\stackrel{\infty}{\infty}} \underset{\infty}{\infty}} &{\text { 广. }} &{\stackrel{m}{\stackrel{m}{m}}} &{\text { 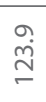 }} &{\stackrel{\infty}{\stackrel{0}{0}}} &{\text { 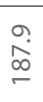 }} &{\text { 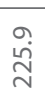 }} &{\begin{array}{l}n ? \\
\stackrel{0}{N}\end{array}} &{\text { 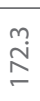 }} &{\underset{\infty}{\underset{\infty}{\infty}}} \\
{\hline} &{\begin{array}{l}\stackrel{u}{\breve{n}} \\
\widetilde{\sigma}\end{array}} &{\text { 割 }} &{\hat{o}} &{\text { 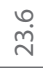 }} &{\stackrel{m}{\stackrel{m}{ }}} &{\text { กุ }} &{\text { nn }} &{\stackrel{ナ}{\stackrel{\oplus}{\infty}}} &{\begin{array}{c}m \\
\stackrel{n}{n}\end{array}} &{\text { 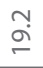 }} &{\text { જิ }} &{\text { ஸ் }} &{\stackrel{\text { ㄴ? }}{\longrightarrow}} &{\text { ळ̊. }} \\
{\hline} &{\text { 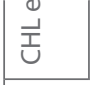 }} &{\text { 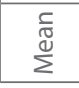 }} &{\begin{array}{l}\stackrel{\infty}{\infty} \\
\stackrel{\infty}{\sim} \\
\sim\end{array}} &{\stackrel{\substack{n \\
\infty}}{\infty}} &{\stackrel{\infty}{\infty}} &{\text { 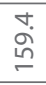 }} &{\frac{m}{m}} &{\begin{array}{l}\stackrel{t}{6} \\
\stackrel{0}{=}\end{array}} &{\text { 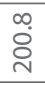 }} &{\begin{array}{l}\infty \\
\stackrel{\infty}{\infty} \\
\infty\end{array}} &{\stackrel{\bar{\infty}}{\stackrel{\sim}{N}}} &{\text { 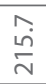 }} &{\stackrel{N}{\stackrel{N}{N}}} &{\text { ণ্் }} \\
{\hline} &{ } &{ } &{\stackrel{\sim}{\sim}} &{\text { ָָ }} &{\hat{\mathrm{i}}} &{\stackrel{\sim}{r}} &{N} &{\bar{i}} &{\cong} &{\stackrel{\infty}{-}} &{\stackrel{m}{r}} &{\stackrel{?}{-}} &{\stackrel{\bullet}{\sim}} &{\text { 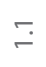 }} \\
{\hline} &{\text { 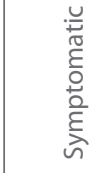 }} &{\frac{\frac{\bar{d}}{0}}{\frac{0}{3}}} &{\lrcorner} &{\text { 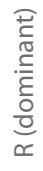 }} &{\lrcorner} &{\lrcorner} &{\lrcorner} &{-} &{\text { 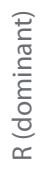 }} &{\text { 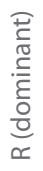 }} &{-} &{-} &{-} &{\simeq} \\
{\hline} &{ } &{ } &{\stackrel{\infty}{\sim}} &{\bar{\tau}} &{\stackrel{\sim}{*}} &{\text { ㅇํ }} &{\text { กิ }} &{\text { nn }} &{\text { นก }} &{\text { 织 }} &{\text { ㄴำ }} &{\stackrel{\infty}{\infty}} &{\tilde{\sigma}} &{\mathbb{N}} \\
$\hline
\end{tabular}


David McKean, Siok Li Chung, Rebecca te Water Naudé, Bernard McElroy, Jonathan Baxter,

Aniruddha Pendse, Joseph Papanikitas, James Teh, Richard Hughes

Tab. 4. Comparison of paired measurements of coracohumeral ligament (CHL) elasticity of the symptomatic shoulder pre- and post-treatment in the neutral and $30^{\circ} \mathrm{ER}$ position

\begin{tabular}{|c|c|c|c|}
\hline Parameter & Pre-injection $(n=8)$ & Post-injection $(n=8)$ & Pvalue ${ }^{\ddagger}$ \\
\hline CHL thickness (mm) & $3.2(2.4-3.7)^{*}$ & $2.5(1.9-3.2)^{*}$ & $<0.05$ \\
\hline \multicolumn{4}{|l|}{ CHL elastic modulus (kPa) } \\
\hline Neutral position & $286.4(208.9-338.4)^{*}$ & $226.5(179.4-265.8)^{*}$ & $<0.05$ \\
\hline $30^{\circ}$ external rotation ${ }^{\dagger}$ & $303.7(221.4-342.2)^{*}$ & $245.0(197.7-273.2)^{*}$ & $<0.05$ \\
\hline OSS & $13.5(7-28)^{*}$ & $34(22-46)^{*}$ & $<0.05$ \\
\hline \multirow{3}{*}{ VAS pain score } & \multirow{3}{*}{$8.5(6-10)^{*}$} & $3.5(0-10) *$ at 24 hours & $<0.05$ \\
\hline & & $2(0-5)^{*}$ at 1 week & $<0.05$ \\
\hline & & $2.5(1-6)^{*}$ at 8 weeks & $<0.05$ \\
\hline
\end{tabular}

anaesthetic block. Follow-up assessment of the elasticity of the CHL was obtained in 8 treated patients $(3$ men, 5 women, median age 55.5 years, range $41-72$ years) at 10 weeks post-injection. Treated patients had an excellent response to ultrasound guided rotator interval injection and manipulation under local anaesthetic block, with improved OSS and reduction in VAS pain scores. Median OSS was 13.5 pre-injection and 34 at 10 weeks post-injection. Median VAS pain scores measured 8.5 preinjection, 3.5 at 1 day, 2 at 1 week, and 2.5 at 10 weeks. This was associated with a trend to normalisation of the elastic modus of the treated shoulder measured in the neutral position (mean $225 \mathrm{kPa}$ at 10 weeks post-injection, range $179-266 \mathrm{kPa}$, median $226.5 \mathrm{kPa}$ ) and at to $30^{\circ}$ of external rotation (mean $236.7 \mathrm{kPa}$ at 10 weeks postinjection, range $198-273 \mathrm{kPa}$, median $245.0 \mathrm{kPa}$ ). (Tab. 4 and Tab. 5).

\section{Discussion}

Using SWE, we found that the CHL was significantly thicker and stiffer in the symptomatic shoulder compared with the asymptomatic side, consistent with previous studies $^{(13,14)}$. Previous studies have stated that the thickening of the CHL is a key imaging finding in adhesive capsulitis on $\mathrm{US}^{(6)}$ and MR imaging ${ }^{(10,21-23)}$. This is consistent with the hypothesis that stiffening of the CHL may play a key role in limiting the range of movement in frozen shoulder ${ }^{(13,14)}$. To date, there was no available data obtained using SWE regarding the elasticity of the CHL post-treatment for frozen shoulder. In this study, we observed a reduction in the stiffness of the CHL in both a neutral position and maximal external rotation following successful treatment with ultrasound guided rotator interval injection. This reduction in CHL stiffness was associated with reduced pain scores and improved Oxford Shoulder Scores. This clinical improvement is consistent with previous papers describing rotator interval injections for the treatment of adhesive capsulitis ${ }^{(24-26)}$. These findings may support the previous orthopaedic investigation that release of the CHL is associated with increased shoulder $\operatorname{ROM}^{(5,11,12)}$. Several other biomechanical studies have also indicated that tension in the CHL can have a significant impact on glenohumeral joint range of movement by providing resistance in the inferior and posterior translations of the humeral head and resisting rotation ${ }^{(27-29)}$. A number of different treatments are available for adhesive capsulitis including physiotherapy, steroid injections, hydrodilatation, manipulation under anaesthesia, and arthroscopic capsular release $\mathrm{e}^{(1,30)}$. The patients in our study were only treated with rotator interval injections and manipulation under local anaesthesia. The authors speculate that manipulation under local anaesthesia may allow for an improved range of movement by directly stretching the stiffened CHL and capsule. All our patients are advised to perform regular exercises to maintain their range of movement, and the authors postulate that continued exercise with associated progressive improvement in range of movement likely contributes to the changes in elasticity described in our paper, however further studies are required to investigate this further. In addition, future research is required to determine whether other treatment strategies may result in similar normalisation of CHL elasticity.

There are several limitations to our study which should be addressed. None of our patients underwent arthroscopic release, and no histological correlation is available. Our study cohort is relatively small, and larger age- and sex-matched studies would provide additional information. Differences in measurement locations could have a significant impact on SWE measurements, and it is possible that measurements taken closer to the attachment site may result in higher CHL elasticity measurements. While our study protocol was consistent in the location of measurement, only one region of interest was analysed near the coracoid process. This was also consistent with previous published work $^{(13)}$, but may not provide a complete picture of the elastic properties of the entire CHL. Other recent studies have suggested that changes in the elastic modulus of the rotator cuff tendons may also occur in adhesive capsulitis ${ }^{(17,31)}$, which we have not measured. Further studies are required to further investigate these findings in order to fully understand complex changes in the elastic properties of the rotator interval structures and rotator cuff tendons that may occur in adhesive capsulitis. However, we believe that these limitations did not affect our results. 
Tab. 5. Coracohumeral ligament (CHL) thickness and elasticity of the symptomatic shoulder pre- and post-treatment in the neutral and $30^{\circ}$ external rotation (ER) position

\begin{tabular}{|c|c|c|c|c|c|c|c|c|c|c|}
\hline \multirow{11}{*}{ 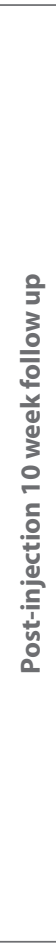 } & \multirow{5}{*}{ 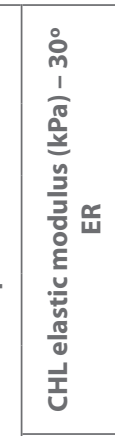 } & 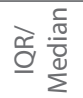 & $\stackrel{N}{\circ}$ & $\stackrel{\sim}{\circ}$ & $\overline{ָ ָ}$ & $\begin{array}{l}\text { ö } \\
\text { Oे }\end{array}$ & $\stackrel{\nwarrow}{\circ}$ & $\stackrel{8}{0}$ & $\stackrel{n}{\circ}$ & $\frac{\infty}{0}$ \\
\hline & & $\underline{\widetilde{\sigma}}$ & $\begin{array}{l}\stackrel{0}{0} \\
\stackrel{N}{0}\end{array}$ & 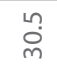 & $\begin{array}{l}\infty \\
\text { గi } \\
\end{array}$ & ఫే & 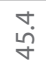 & $\stackrel{+}{=}$ & $m$ & $\stackrel{\substack{m \\
m}}{m}$ \\
\hline & & 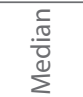 & $\stackrel{\bar{\infty}}{\stackrel{\infty}{\sim}}$ & $\stackrel{\mathcal{J}}{\mathrm{J}}$ & 号 & $\stackrel{\text { }}{\sim}$ & 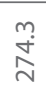 & $\stackrel{m}{\stackrel{m}{a}}$ & $\stackrel{0}{\stackrel{\infty}{\infty}}$ & $\underset{\substack{\downarrow \\
\infty}}{\stackrel{\nabla}{\infty}}$ \\
\hline & & in & $\stackrel{\sim}{\stackrel{n}{\sim}}$ & $\stackrel{\stackrel{n}{\alpha}}{\sigma}$ & $\stackrel{\text { m }}{ }$ & $\stackrel{\circ}{\sim}$ & $\stackrel{+}{\sigma}$ & $\stackrel{\infty}{\underset{\sim}{+}}$ & 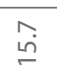 & $\stackrel{m}{n}$ \\
\hline & & 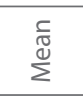 & $\stackrel{\stackrel{\sim}{\sim}}{\underset{\sim}{\sim}}$ & 吕 & 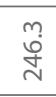 & $\stackrel{N}{\stackrel{N}{N}}$ & - & $\begin{array}{l}\stackrel{\leftrightarrow}{\infty} \\
\stackrel{\sigma}{\circ}\end{array}$ & $\stackrel{\stackrel{\sim}{\sim}}{\stackrel{N}{N}}$ & $\hat{\hat{a}}$ \\
\hline & \multirow{5}{*}{ 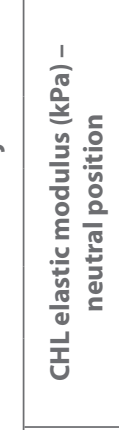 } & 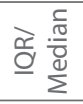 & o̊. & $\stackrel{0}{\circ}$ & $\stackrel{\infty}{0}$ & $\overline{0}$ & $\stackrel{\infty}{\circ}$ & $\overline{0}$ & $\stackrel{\sim}{\sim}$ & ô. \\
\hline & & $\underline{\widetilde{\alpha}}$ & $\hat{\text { iิ }}$ & 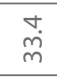 & $\begin{array}{l}\stackrel{\rho}{\infty} \\
\stackrel{\infty}{\sigma}\end{array}$ & ָ̃. & $\stackrel{m}{i}$ & $\bar{\sim}$ & in & $\stackrel{\sim}{\underline{m}}$ \\
\hline & & 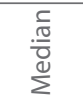 & $\begin{array}{l}\stackrel{n}{0} \\
\infty \\
\sim \\
\sim\end{array}$ & $\stackrel{+}{\stackrel{\circ}{N}}$ & ָ̃ & 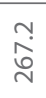 & $\begin{array}{l}\infty \\
\infty \\
\infty \\
\stackrel{0}{N}\end{array}$ & $\begin{array}{l}\alpha \\
\sigma \\
\sigma\end{array}$ & 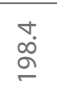 & $\underset{\substack{\sim \\
\infty}}{\sim}$ \\
\hline & & 只 & $\begin{array}{l}L_{\infty} \\
\infty\end{array}$ & $\stackrel{\text { Ĩ }}{\leftarrow}$ & $\hat{\imath}$ & 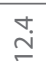 & สี & $\hat{\stackrel{m}{m}}$ & $\stackrel{\sim}{\sim}$ & î \\
\hline & & 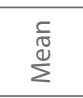 & $\stackrel{\widehat{N}}{\sim}$ & $\stackrel{\circ}{\sim}$ & ঐे. & $\begin{array}{l}\infty \\
\text { î. } \\
\text { î. }\end{array}$ & $\begin{array}{l}\text { ? } \\
\stackrel{+}{0} \\
\text { N }\end{array}$ & $\stackrel{\sim}{\infty}$ & 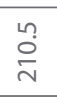 & ז. \\
\hline & 로 & & $\stackrel{?}{-}$ & $\stackrel{\stackrel{\sim}{i}}{ }$ & $\stackrel{\circ}{\text { i }}$ & $\stackrel{\sim}{m}$ & $m$ & $\stackrel{m}{i}$ & $\bar{i}$ & $\stackrel{\leftrightarrow}{\leftrightarrow}$ \\
\hline \multirow{12}{*}{ 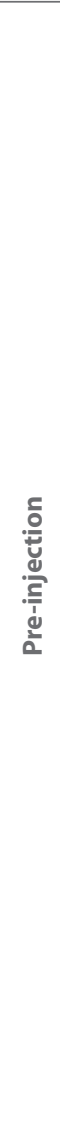 } & \multirow{5}{*}{ 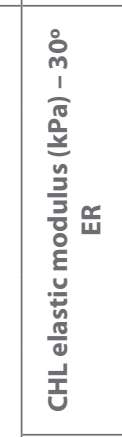 } & 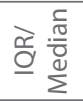 & ¿̊. & $\stackrel{\tilde{O}}{0}$ & $\stackrel{m}{o}$ & oे & t̊. & ộ. & ơ & $\stackrel{\circ}{\circ}$ \\
\hline & & $\underline{q}$ & $\stackrel{\infty}{0}^{\circ}$ & $\stackrel{\infty}{\wedge}$ & $\stackrel{\circ}{\infty}$ & ฌ્ & 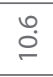 & $\stackrel{n}{\stackrel{n}{N}}$ & $\stackrel{m}{+}$ & 广̊․ \\
\hline & & 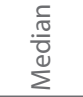 & 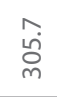 & $\stackrel{m}{m}$ & $\stackrel{\stackrel{n}{m}}{m}$ & 吕 & $\begin{array}{l}\text { வீ. } \\
\text { வे }\end{array}$ & $\begin{array}{l}m \\
\mathscr{o}^{\circ} \\
m\end{array}$ & $\stackrel{\infty}{\stackrel{\sim}{0}}$ & $\begin{array}{l}\text { gे } \\
\dot{g} \\
\text { d }\end{array}$ \\
\hline & & 只 & $\begin{array}{l}\text { ָे } \\
\text { ஸे } \\
\end{array}$ & $\begin{array}{l}\text { 员 } \\
\text { hn } \\
\end{array}$ & i̊n & $\begin{array}{l}\stackrel{9}{9} \\
0 \\
0\end{array}$ & $\stackrel{\text { nq }}{q}$ & $\begin{array}{l}q \\
\infty \\
\infty\end{array}$ & 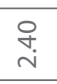 & 움 \\
\hline & & $\sum_{\substack{\mathbb{\sigma} \\
\Sigma}}$ & $\hat{⿱ ⺈ ⿵ ⺆ ⿻ 二 丨 冂 口 ⿱ ~}$ & $\begin{array}{l}\text { ò } \\
\dot{j} \\
\text { jo }\end{array}$ & $\stackrel{\circ}{\stackrel{m}{m}}$ & 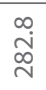 & ্ָণ & $\begin{array}{l}0 \\
\stackrel{\rho}{\text { p. }}\end{array}$ & 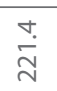 & $\underset{\mathcal{H}}{\tilde{H}}$ \\
\hline & \multirow{5}{*}{ 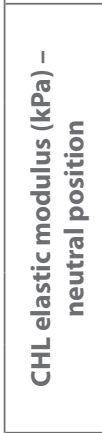 } & 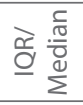 & $\overline{0}$ & ¿̊. & O্. & $\stackrel{1}{0}$ & ¿̊. & ঙํ & ò. & $\frac{\infty}{0}$ \\
\hline & & $\stackrel{\mathscr{0}}{\underline{O}}$ & 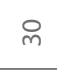 & $\stackrel{0}{0}$ & 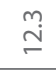 & gे & $\stackrel{\circ}{\circ}$ & $\hat{0}$ & $\stackrel{\circ}{\circ}$ & 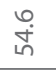 \\
\hline & & 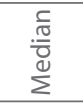 & $\begin{array}{l}\infty \\
\infty \\
\stackrel{\infty}{\sim} \\
\end{array}$ & 이 & 於 & 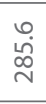 & 迥 & $\frac{m}{\grave{n}}$ & $\stackrel{m}{\stackrel{m}{\sim}}$ & $\begin{array}{l}\text { O. } \\
\stackrel{0}{m}\end{array}$ \\
\hline & & 虽 & $\stackrel{m}{\sim}$ & 㝒 & $\hat{\sigma}$ & $\stackrel{\infty}{\stackrel{\sim}{N}}$ & $\stackrel{n}{\leftarrow}$ & $\begin{array}{l}\infty \\
\stackrel{\infty}{\sim}\end{array}$ & $\bar{\infty}_{\infty}^{-}$ & 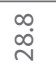 \\
\hline & & 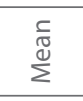 & ণ্ণ & $\stackrel{\sim}{\stackrel{0}{m}}$ & $\begin{array}{l}+ \\
\infty \\
m \\
m\end{array}$ & 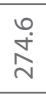 & ্ָণ & 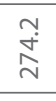 & $\begin{array}{l}\text { aे } \\
\infty \\
\stackrel{\text { N }}{ }\end{array}$ & $\begin{array}{l}\text { o. } \\
\dot{0} \\
\tilde{m}\end{array}$ \\
\hline & 悹 $\overline{\underline{\xi}}$ & & $m$ & $\hat{m}$ & $\hat{m}$ & $m$ & $m$ & $\bar{m}$ & $\stackrel{\leftrightarrow}{m}$ & $\stackrel{m}{m}$ \\
\hline & 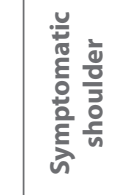 & & 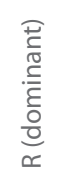 & \lrcorner & \lrcorner & 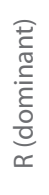 & \lrcorner & \lrcorner & \lrcorner & $\propto$ \\
\hline & ஓ & & $\bar{\gamma}$ & ก & $\stackrel{n}{n}$ & 㔯 & in & $\stackrel{\infty}{n}$ & $\hat{\sigma}$ & $N$ \\
\hline
\end{tabular}

\section{Conclusion}

US guided injection of the rotator interval and manipulation under local anaesthetic block was associated with a reduction in CHL stiffness and significantly improved patient symptoms. This is the first data describing changes in CHL stiffness changes following successful treatment for adhesive capsulitis and associated improved range of movement. This finding supports previous work that has implicated changes in CHL stiffness as a key structure in restricting range of glenohumeral joint motion in patients with adhesive capsulitis. Further research is needed to fully evaluate the pathological changes in the elasticity of the shoulder soft tissues that may occur in adhesive capsulitis and how these may improve.

\section{Conflict of interest}

Authors do not report any financial or personal connections with other persons or organisations, which might negatively affect the contents of this publication and/or claim authorship rights to this publication. 


\section{References}

1. Neviaser AS, Hannafin JA: Adhesive capsulitis: a review of current treatment. Am J Sports Med 2010; 38: 2346-2356.

2. Hsu JE, Anakwenze OA, Warrender WJ, Abboud JA: Current review of adhesive capsulitis. J Shoulder Elbow Surg 2011; 20: 502-514.

3. Hand GCR, Athanasou NA, Matthews T, Carr AJ: The pathology of frozen shoulder. J Bone Joint Surg Br 2007; 89: 928-932.

4. Neviaser JS. Adhesive capsulitis of the shoulder (the frozen shoulder). Med Times 1962; 90: 783-807.

5. Ozaki J, Nakagawa Y, Sakurai G, Tamai S: Recalcitrant chronic adhesive capsulitis of the shoulder. Role of contracture of the coracohumeral ligament and rotator interval in pathogenesis and treatment. J Bone Joint Surg Am 1989; 71: 1511-1515.

6. Homsi C, Bordalo-Rodrigues M, da Silva JJ, Stump XMGRG: Ultrasound in adhesive capsulitis of the shoulder: is assessment of the coracohumeral ligament a valuable diagnostic tool? Skeletal Radiol 2006; 35: 673-678.

7. Rundquist PJ, Anderson DD, Guanche CA, Ludewig PM: Shoulder kinematics in subjects with frozen shoulder. Arch Phys Med Rehabil 2003; 84: 1473-1479.

8. Neer CS 2nd, Satterlee CC, Dalsey RM, Flatow EL: The anatomy and potential effects of contracture of the coracohumeral ligament. Clin Orthop Relat Res 1992; (280): 182-185.

9. Kanazawa K, Hagiwara Y, Kawai N, Sekiguchi T, Koide M, Ando A et al:: Correlations of coracohumeral ligament and range of motion restriction in patients with recurrent anterior glenohumeral instability evaluated by magnetic resonance arthrography. J Shoulder Elbow Surg 2017; 26: 233-240.

10. Lee S-Y, Park J, Song S-W: Correlation of MR arthrographic findings and range of shoulder motions in patients with frozen shoulder. AJR Am J Roentgenol 2012; 198: 173-179.

11. Hagiwara Y, Ando A, Kanazawa K, Koide M, Sekiguchi T, Hamada J et al.: Arthroscopic coracohumeral ligament release for patients with frozen shoulder. Arthrosc Tech 2018; 7: e1-e5.

12. Eid A: Miniopen coracohumeral ligament release and manipulation for idiopathic frozen shoulder. Int J Shoulder Surg 2012; 6: 90-96.

13. Wu C-H, Chen W-S, Wang T-G: Elasticity of the coracohumeral ligament in patients with adhesive capsulitis of the shoulder. Radiology 2016; 278 : 458-464.

14. Kanazawa K, Hagiwara Y, Sekiguchi T, Fujita R, Suzuki K, Koide M et al.: Elastic changes of the coracohumeral ligament evaluated with shear wave elastography. Open Orthop J 2018; 12: 427-434.

15. Kanazawa K, Hagiwara Y, Sekiguchi T, Fujita R, Suzuki K, Koide M et al.: Correlations between range of motion and elasticity of the coracohumeral ligament evaluated with shear-wave elastography. J Sport Rehabil 2020; 1-7.

16. Rosskopf AB, Ehrmann C, Buck FM, Gerber C, Flück M, Pfirrmann CWA: Quantitative shear-wave US elastography of the supraspinatus muscle: reli- ability of the method and relation to tendon integrity and muscle quality. Radiology 2016; 278: 465-474.

17. Wada T, Itoigawa Y, Yoshida K, Kawasaki T, Maruyama Y, Kaneko K: Increased stiffness of rotator cuff tendons in frozen shoulder on shear wave elastography. J Ultrasound Med 2020; 39: 89-97.

18. Zhu B, You Y, Xiang X, Wang L, Qiu L: Assessment of common extensor tendon elasticity in patients with lateral epicondylitis using shear wave elastography. Quant Imaging Med Surg 2020; 10: 211-219.

19. De Zordo T, Fink C, Feuchtner GM, Smekal V, Reindl M, Klauser AS: Realtime sonoelastography findings in healthy Achilles tendons. AJR Am J Roentgenol 2009; 193: W134-W138.

20. Dawson J, Fitzpatrick R, Carr A: Questionnaire on the perceptions of patients about shoulder surgery. J Bone Joint Surg Br 1996; 78: 593-600.

21. Li J, Tang K, Wang J, Li Q, Xu H, Yang H et al.: MRI findings for frozen shoulder evaluation: is the thickness of the coracohumeral ligament a valuable diagnostic tool? PLoS One 2011; 6: e28704.

22. Mengiardi B, Pfirrmann CWA, Gerber C, Hodler J, Zanetti M: Frozen shoulder: MR arthrographic findings. Radiology 2004; 233: 486-492.

23. Zhao W, Zheng X, Liu Y, Yang W, Amirbekian V, Diaz LE et al.: An MRI study of symptomatic adhesive capsulitis. PLoS One 2012; 7: e47277.

24. McKean D, Yoong P, Brooks R, Papanikitas J, Hughes R, Pendse A et al:: Shoulder manipulation under targeted ultrasound-guided rotator interval block for adhesive capsulitis. Skeletal Radiol 2019; 48: 1269-1274.

25. Yoong P, Duffy S, McKean D, Hujairi NP, Mansour R, Teh JL: Targeted ultrasound-guided hydrodilatation via the rotator interval for adhesive capsulitis. Skeletal Radiol 2015; 44: 703-708.

26. Prestgaard T, Wormgoor MEA, Haugen S, Harstad H, Mowinckel P, Brox JI: Ultrasound-guided intra-articular and rotator interval corticosteroid injections in adhesive capsulitis of the shoulder: a double-blind, sham-controlled randomized study. Pain 2015; 156: 1683-1691.

27. Harryman DT, Sidles JA, Harris SL, Matsen FA: The role of the rotator interval capsule in passive motion and stability of the shoulder. J Bone Joint Surg Am 1992; 74: 53-66.

28. Boardman ND, Debski RE, Warner JJP, Taskiran E, Maddox L, Imhoff AB et al:: Tensile properties of the superior glenohumeral and coracohumeral ligaments. J Shoulder Elbow Surg 1996; 5: 249-254.

29. Ovesen J, Nielsen S: Experimental distal subluxation in the glenohumeral joint. Arch Orthop Trauma Surg 1985; 104: 78-81.

30. Cho C-H, Bae K-C, Kim D-H: Treatment strategy for frozen shoulder. Clin Orthop Surg 2019; 11: 249-257.

31. Yun SJ, Jin W, Cho NS, Ryu KN, Yoon YC, Cha JG et al.: Shear-wave and strain ultrasound elastography of the supraspinatus and infraspinatus tendons in patients with idiopathic adhesive capsulitis of the shoulder: a prospective case-control study. Korean J Radiol 2019; 20: 1176-1185. 